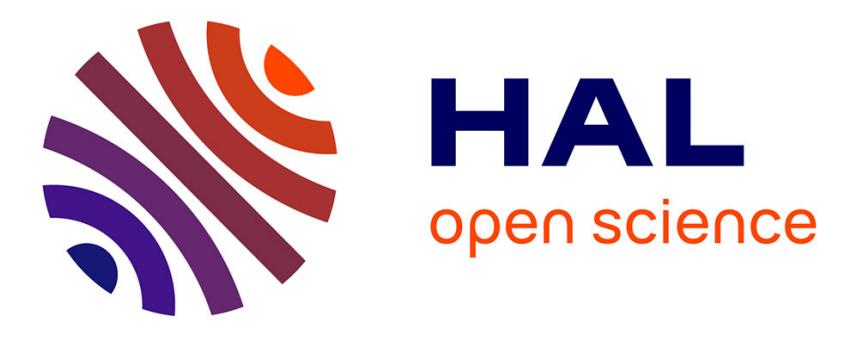

\title{
Calibrationless oscar-based image reconstruction in compressed sensing parallel MRI
}

Loubna El Gueddari, Philippe Ciuciu, Emilie Chouzenoux, Alexandre

Vignaud, Jean-Christophe Pesquet

\section{- To cite this version:}

Loubna El Gueddari, Philippe Ciuciu, Emilie Chouzenoux, Alexandre Vignaud, Jean-Christophe Pesquet. Calibrationless oscar-based image reconstruction in compressed sensing parallel MRI. ISBI 2019 - IEEE International Symposium on Biomedical Imaging, Apr 2019, Venise, Italy. 10.1109/isbi.2019.8759393 . hal-02101262

\section{HAL Id: hal-02101262 \\ https://hal.inria.fr/hal-02101262}

Submitted on 16 Apr 2019

HAL is a multi-disciplinary open access archive for the deposit and dissemination of scientific research documents, whether they are published or not. The documents may come from teaching and research institutions in France or abroad, or from public or private research centers.
L'archive ouverte pluridisciplinaire HAL, est destinée au dépôt et à la diffusion de documents scientifiques de niveau recherche, publiés ou non, émanant des établissements d'enseignement et de recherche français ou étrangers, des laboratoires publics ou privés. 


\title{
CALIBRATIONLESS OSCAR-BASED IMAGE RECONSTRUCTION IN COMPRESSED SENSING PARALLEL MRI
}

\author{
L. El Gueddari ${ }^{(1,2)}$, P. Ciuciu ${ }^{(1,2)}$, E. Chouzenoux $^{(3,4)}$, A. Vignaud $^{(1)}$ and J.-C. Pesquet $^{(3)}$ \\ ${ }^{(1)}$ CEA/NeuroSpin, Bât 145, F-91191 Gif-sur Yvette, France. \\ ${ }^{(2)}$ INRIA-CEA Saclay Ile-de-France, Parietal team, Univ. Paris-Saclay, France. \\ (3) CVN, Centrale-Supélec, Univ. Paris-Saclay, France. \\ (4) LIGM, Paris-Est University, France.
}

\begin{abstract}
Reducing acquisition time is a crucial issue in MRI especially in the high resolution context. Compressed sensing has faced this problem for a decade. However, to maintain a high signal-to-noise ratio (SNR), CS must be combined with parallel imaging. This leads to harder reconstruction problems that usually require the knowledge of coil sensitivity profiles. In this work, we introduce a calibrationless image reconstruction approach that no longer requires this knowledge. The originality of this work lies in using for reconstruction a group sparsity structure (called OSCAR) across channels that handles SNR inhomogeneities across receivers. We compare this reconstruction with other calibrationless approaches based on groupLASSO and its sparse variation as well as with the auto-calibrated method called $\ell_{1}$-ESPIRiT. We demonstrate that OSCAR outperforms its competitors and provides similar results to $\ell_{1}$-ESPIRiT. This suggests that the sensitivity maps are no longer required to perform combined CS and parallel imaging reconstruction.
\end{abstract}

Index Terms - Compressed Sensing, Parallel MRI, Group Sparsity, Proximal algorithm

\section{INTRODUCTION}

Context. Magnetic resonance imaging (MRI) is a powerful tool to non-invasively probe soft-tissues. However, MRI suffers from long acquisition time, especially for $\mathrm{T} 2 *$-weighted high-resolution imaging where both long repetition time and echo time hold. Compressed Sensing (CS) has been proposed to shorten scan time by collecting fewer measurements in the Fourier domain or k-space [1, 2]. This theory relies on three ingredients: (i) the existence of a sparse or compressible representation of images; (ii) the ability to design incoherent sampling schemes with respect to this sparse representation and finally (iii) nonlinear reconstruction that promotes sparsity such as $\ell_{1}$ minimization under data consistency constraints. CS has been successfully applied to MRI [3], first using Poisson-Disk sampling and then considering more efficient non-Cartesian trajectories (e.g. spirals, radial [4], Sparkling [5, 6] ...) that allow to break down the coherence barrier [7,8] using 2D variable density sampling $[9,8]$. The resulting acceleration factor (e.g. 6 to 10) is larger than those resulting from standard parallel imaging (PI) acceleration methods. However, for a given image resolution, the maximum acceleration factor depends on the input Signal-to-Noise Ratio (SNR) [10]. Therefore, to ensure a certain level of SNR, CS has been combined with multiple receiver coils (e.g. parallel imaging or PI [11]). In this context, many algorithms have been proposed to reconstruct MR images from subsampled measurements collected over multichannel coils. Those algorithms can be split into four different categories.

Related works. The first class formulates the problem in the original space, the k-space. Most of those methods are based on the minimization of an inconsistency term between a region of Auto-Calibrating Signal (ACS region), where the signal is acquired respecting the Nyquist-Shannon criteria and the missing samples which are estimated as in Generalized Auto-calibration Partially Parallel Acquisitions GRAPPA method [12, 13]. Other recent methods impose low-rank constraints in the k-space such as LORAKS [14], they have been successfully applied to MR reconstruction. In general, k-space based reconstructions have the advantage to be more robust to motion. However, their generalization to non-Cartesian sampling schemes is more challenging and implies the use of gridding operator. However, this can be computationally demanding and degrades the image quality. The second class of methods is model-based and tries to model the spatial encoding of the channels sensitivity (SENSE) [15] to recover a single image where the channels' reception profiles have been eliminated. The CS-SENSE reconstruction methods have been introduced in [16] and further extended to non-Cartesian sampling in [17]. Those methods can include correction to off-resonance artifacts, but they require explicit knowledge of sensitivity matrices. To estimate the latter, two kinds of approaches have been proposed: first, blind methods estimate both sensitivity profiles and the unknown MR image to be reconstructed [12, 18, 19]. Second, self-calibrating sequential approaches first extract sensitivity profiles using a k-space region where the measurements have been fully collected or at least a heavily sampled region ( $\mathrm{k}$-space center) [20, 21, 22]. The third category is a hybrid version between SENSE and GRAPPA-like methods characterized by an inconsistency term between the ACS region and the explicit knowledge of sensitivity matrices (e.g. PRUNO [23] or $\ell_{1}$ ESPIRiT [21]). Finally, the last class of methods is calibrationless and characterized by the use of joint sparsity. The reconstruction problem is solved as an inverse problem [24, 25]. The latter presents the advantage of avoiding the acquisition of the ACS region and estimation of sensitivity matrices. Such methods have improved recovery guarantees compared to classical CS model as demonstrated in $[26,27,28]$.

Goals and contributions. In this work, we will focus on the last class and we will compare three different regularization terms that promote joint sparsity: the group-LASSO [24], sparse groupLASSO [29], and Octagonal Shrinkage and Clustering Algorithm for Regression (OSCAR) [30]. To the best of our knowledge, it is the first time that OSCAR penalization has been applied in to a reconstruction problem. Moreover, we rely on a generic primal- 
dual optimization algorithm that solves all penalizations in a unified framework. Our results on ex vivo baboon brain data collected at 7 Tesla demonstrate that calibration-less reconstruction methods may achieve the same image quality as (or slightly better than) auto-calibrated ones.

\section{PROBLEM STATEMENT}

Variational formulation. Let $N$ be the resolution of the recovered image per channel, let $L$ be the number of channels used to acquire the NMR signal, and let $M$ be the number of measurements in the Fourier domain with $M<N$. We denote by $\boldsymbol{y} \in \mathbb{C}^{M \times L}$ the acquired NMR signal and by $\boldsymbol{y}_{\ell} \in \mathbb{C}^{M}$ the $\ell^{\text {th }}$ channel-specific dataset. Let $\underline{\boldsymbol{x}}=\left[\boldsymbol{x}_{1}, \ldots, \boldsymbol{x}_{L}\right] \in \mathbb{C}^{N \times L}$ be the reconstructed MR images such that $\boldsymbol{x}_{\ell} \in \mathbb{C}^{N}$ is associated with the $\ell^{\text {th }}$ channel. The image reconstruction problem reads as follows:

$$
\underline{\widehat{\boldsymbol{x}}}=\underset{\underline{\boldsymbol{x}} \in \mathbb{C}^{N \times L}}{\arg \min }\left\{\frac{1}{2} \sum_{\ell=1}^{L} \sigma_{\ell}^{-2}\left\|f_{\Omega}\left(\boldsymbol{x}_{\ell}\right)-\boldsymbol{y}_{\ell}\right\|_{2}^{2}+g(\boldsymbol{T} \underline{\boldsymbol{x}})\right\},
$$

where $f_{\Omega}$ is the forward under-sampling Fourier operator. In the case of Cartesian sampling, $f_{\Omega}\left(\boldsymbol{x}_{\ell}\right)=\boldsymbol{\Omega} \boldsymbol{F} \boldsymbol{x}_{\ell}$ with $\boldsymbol{F}$ the 2D fast Fourier transform (FFT) and $\boldsymbol{\Omega}$ the binary under-sampling mask defined over the discrete grid. In non-Cartesian settings, $f_{\Omega}$ refers to nonequispaced or nonuniform FFT [31, 32] and $\Omega$ stands for the continuous support of the measurements in k-space. $\boldsymbol{T} \in \mathbb{C}^{N_{\Psi} \times N}$ is a linear operator related to a multiscale decomposition $\Psi$ with output size $N_{\Psi}$ (e.g., wavelet transform). Let $\Gamma_{0}\left(\mathbb{C}^{N_{\Psi} \times L}\right)$ be the set of convex, proper, lower semi-continuous functions on $\mathbb{C}^{N_{\Psi} \times L}$ that take values in $\mathbb{R} \cup\{+\infty\}$. Function $g \in \Gamma_{0}\left(\mathbb{C}^{N_{\Psi} \times L}\right)$ is the regularization term that promotes sparsity of the wavelet coefficients across channels. This formulation enables the use of over-complete dictionaries as will be done in this work. In this work, we will rely on a proximal minimization method and investigate the most suitable choice for $g$. Thus we mainly focus on convex proximal-friendly penalty terms that can be handled using the same generic optimization algorithm.

Primal-dual optimization algorithm. Here we summarize the primal-dual optimization method proposed by Condat-Vũ [33, 34, 35]. We aim to find:

$$
\underline{\widehat{\boldsymbol{x}}} \in \underset{\underline{\boldsymbol{x}} \in \mathbb{C}^{N \times L}}{\operatorname{argmin}}[f(\underline{\boldsymbol{x}})+g(\boldsymbol{T} \underline{\boldsymbol{x}})]
$$

where: (i) $f$ is convex, differentiable on $\mathbb{C}^{N \times L}$ and its gradient is $\beta$-Lipschitz; (ii) $g \in \Gamma_{0}\left(\mathbb{C}^{N_{\Psi} \times L}\right)$ with a closed form proximity operator. We recall that this operator is defined as:

$$
\operatorname{prox}_{g}(\underline{\boldsymbol{z}})=\underset{\boldsymbol{v} \in \mathbb{C}^{N_{\Psi} \times L}}{\operatorname{argmin}} \frac{1}{2}\|\underline{\boldsymbol{z}}-\boldsymbol{v}\|^{2}+g(\boldsymbol{v})
$$

According to [33, Theorem 3.1], Algorithm (1) will converge to a solution of Eq. (2) if $\frac{1}{\tau}-\kappa\|\boldsymbol{T}\|^{2} \geq \frac{\beta}{2}$ where $\|\boldsymbol{T}\|$ is the spectral norm of the $\boldsymbol{T}$ operator

The Lipschitz constant $\beta$ is related to the norm of the linear operator $f_{\Omega}$, namely $\beta=\sum_{\ell=1}^{L} \sigma_{\ell}^{-2}\left\|f_{\Omega}\right\|^{2}$. In practice for Cartesian case $\left\|f_{\Omega}\right\|^{2}=1$ and for non-Cartesian case, we will evaluate the norm by using the power iteration method [36]. Finally, the hyper-parameters in this algorithm are set as follows: $\tau=\frac{1}{\beta}, \kappa=\frac{\beta}{2\|\boldsymbol{T}\|^{2}}$. Note that when $\boldsymbol{T}$ defines an orthonormal basis decomposition, we get $\|\boldsymbol{T}\|=1$.

After convergence, the reconstruction algorithm delivers $\underline{\widehat{x}}$ hence one image per channel. In the end, all coil-specific MR images

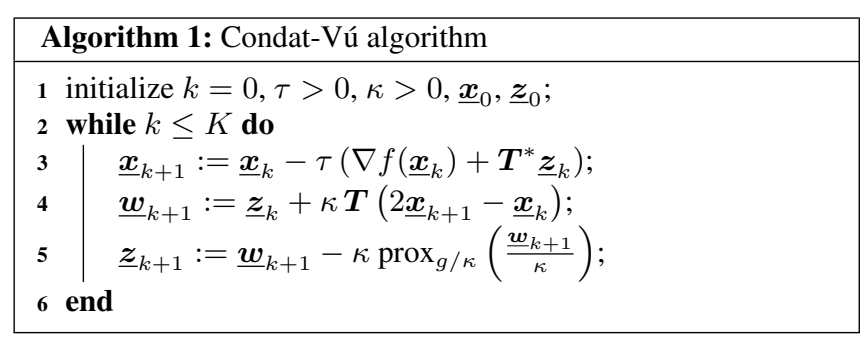

are combined using the square-root of the sum-of-squares (sSOS), $\widehat{\boldsymbol{x}}_{s S O S}=\sqrt{\sum_{\ell=1}^{L}\left\|\widehat{\boldsymbol{x}}_{\ell}\right\|_{2}^{2}}$, to form a single image as usually done in the PI setting.

\section{JOINT-SPARSITY REGULARIZATIONS}

Group-LASSO regularization. The group Least Absolute Shrinkage and Selection Operator or group-LASSO penalty has already been studied in parallel imaging [24]. Moreover, the authors in [26] have proved tighter recovery guarantees (i.e., inequality concentrations) using such regularization term. This means that one can perfectly reconstruct MR images in the noiseless case using a smaller number of measurements collected in parallel imaging as compared to the number required in the single channel mode.

Here, we define $\underline{\boldsymbol{z}}=\left[\boldsymbol{z}_{1}, \ldots, \boldsymbol{z}_{L}\right] \in \mathbb{C}^{N_{\Psi} \times L}$, with $\boldsymbol{z}_{\ell} \in \mathbb{C}^{N_{\Psi}}$ the wavelet coefficients of the $\ell^{\text {th }}$ coil such that $\boldsymbol{z}_{\ell}=\Psi \boldsymbol{x}_{\ell}$ and $\Psi$ being a multiscale wavelet transform with $C$ scales, $S_{c}$ the number of sub-bands in scale $c, P_{s_{c}}$ the number of coefficients in sub-band $s_{c}$, and $N_{\Psi}=\sum_{c=1}^{C} \sum_{s=1}^{S_{c}} P_{s}$. For $\underline{z} \in \mathbb{C}^{N_{\Psi} \times L}$, the group-LASSO regularization is given by:

$$
g_{\mathrm{GL}}(\underline{\boldsymbol{z}})=\|\underline{\boldsymbol{z}}\|_{1,2}=\sum_{c=1}^{C} \lambda \gamma^{c} \sum_{s=1}^{S_{c}}\left(\sum_{p=1}^{P_{s_{c}}} \sqrt{\sum_{\ell=1}^{L}\left|z_{c s p \ell}\right|^{2}}\right)
$$

where $z_{c s p \ell}$ is the $p^{\text {th }}$ wavelet coefficient of the $s^{\text {th }}$ sub-band in the $c^{\text {th }}$ scale for the $\ell^{\text {th }}$ coil. Since the group penalty is separable across scales, sub-bands and inter-sub-band position, the proximity operator of the group-LASSO is separable too and can be treated in parallel. For given $c, s p$ and $l$, the proximity operator reads:

$$
\left(\operatorname{prox}_{\lambda \gamma^{c}\|\cdot\|_{1,2}}(\underline{\boldsymbol{z}})\right)_{c s p \ell}= \begin{cases}z_{c s p \ell}\left(1-\frac{\lambda \gamma^{c}}{\alpha_{c s p}}\right) & , \text { if } \alpha_{c s p} \geq \lambda \gamma^{c} \\ 0 & , \text { otherwise }\end{cases}
$$

with $\alpha_{c s p}=\sqrt{\sum_{\ell=1}^{L}\left|z_{c s p \ell}\right|^{2}}$. Note that the hyper-parameters $\lambda$ and $\gamma$ are both positive and need to be set. $\gamma$ typically enables a scale-dependent regularization according to a power-law behavior [37].

Sparse group-LASSO regularization. While the group sparsity regularization only promotes sparsity across groups, the sparse group-LASSO also tries to impose sparsity within each group (i.e. each scale $c$, sub-band $s$ and position $p$ ). This penalization is defined as follows:

$$
\forall \underline{\boldsymbol{z}} \in \mathbb{C}^{N_{\Psi} \times L}, \quad g_{\mathrm{sGL}}(\underline{\boldsymbol{z}})=g_{\mathrm{GL}}(\underline{\boldsymbol{z}})+\mu\|\underline{\boldsymbol{z}}\|_{1}
$$

The proximity operator is explicit and corresponds to the composition of the proximity operator of the group-LASSO given in Eq. (5) and the soft-thresholding as established in [29]. The hyperparameters that need to be set are $\lambda, \gamma$ and $\mu$, all being positive. 
(a)

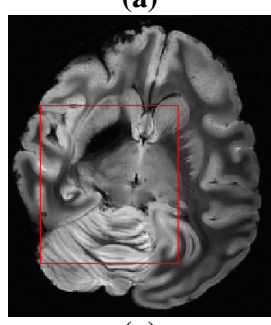

(g)

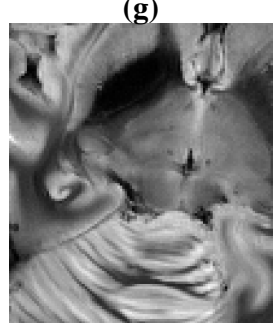

(b)

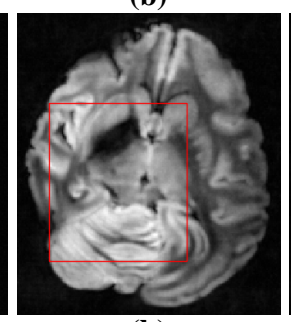

(h)

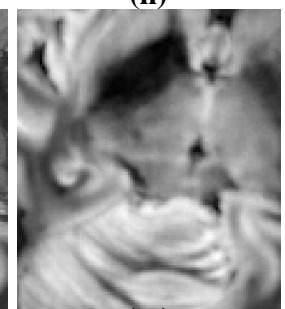

(m)

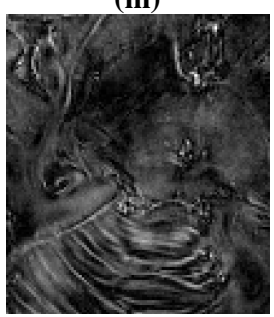

(c)

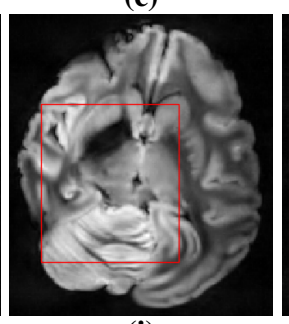

(i)

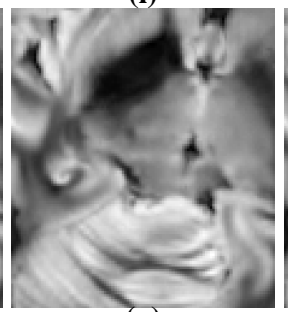

(o)

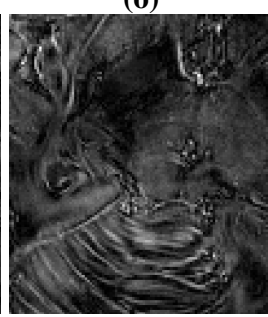

(d)

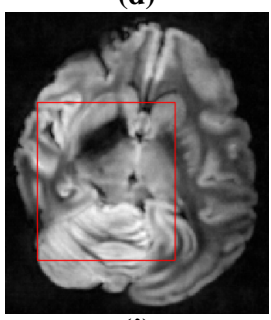

(j)

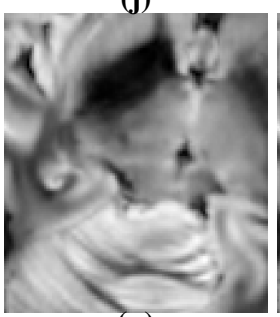

(p)

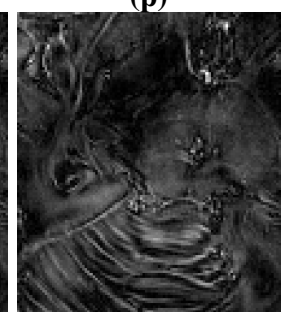

(e)

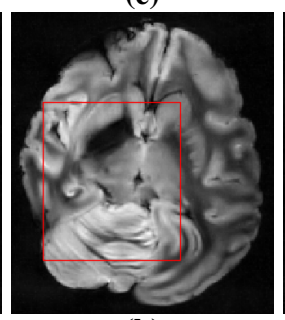

(k)

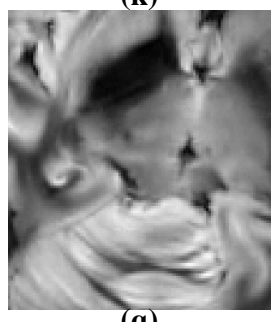

(q)

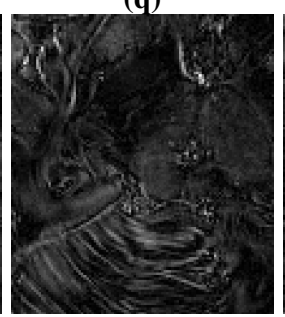

(f)

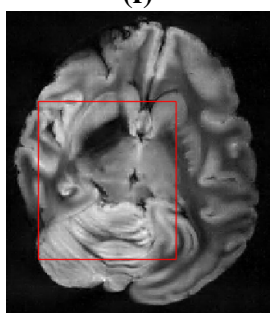

(I)

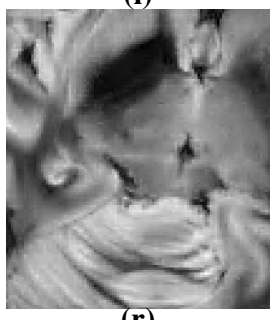

(r)

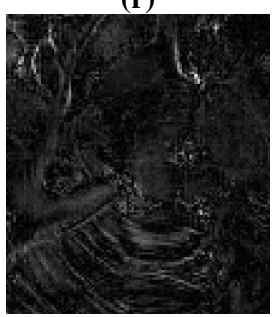

Fig. 1. (a) Cartesian reference. (b) Reconstruction with no regularization term. (c) Reconstruction based on the group-LASSO penalty. (d) Reconstruction based on the sparse group-LASSO penalty. (e) Reconstruction based on OSCAR penalty (f) reconstruction based on $\ell_{1}$-ESPIRiT. (g)-(l) Respective zooms in the red square, (m)-(r) zoom of the difference between the Cartesian referance and the reconstructed image.

OSCAR regularization. In order to go one step further, one may want to add some group-pairing in the regularization, however, inferring the structure is difficult without imposing an explicit prior. To avoid this problem, OSCAR regularization [30] will infer a group structure via a pairwise $\ell_{\infty}$ norm while imposing the $\ell_{1}$ norm as a sparsity constraint. We denote by $\underline{\boldsymbol{z}}_{\downarrow} \in \mathbb{C}^{N_{\Psi} \times L}$ the wavelet coefficients sorted in decreasing order across sub-bands and channels, i.e.: $\forall c \in\{1, \ldots C\}, s \in\left\{1, \ldots, S_{c}\right\}, j \in\left\{1, \ldots, P_{s} L\right\} ;\left|z_{c s 1}\right| \leq$ $\cdots \leq\left|z_{c s j}\right| \leq \cdots \leq\left|z_{c s P_{s} L}\right|$. The OSCAR regularization term reads as follows:

$$
\begin{aligned}
g_{\mathrm{OSCAR}}(\underline{\boldsymbol{z}}) & =\lambda \sum_{c=1}^{C} \sum_{s=1}^{S_{c}}\left[\sum_{j=1}^{P_{s} L}\left(\left|z_{c s j}\right|+\gamma \sum_{k<j} \max \left\{\left|z_{c s j}\right|,\left|z_{c s k}\right|\right\}\right)\right] \\
& =\lambda \sum_{c=1}^{C} \sum_{s=1}^{S_{c}}\left[\sum_{j=1}^{P_{s} L}\left(\gamma\left(P_{s} L-j\right)+1\right)\left|\left(\underline{\boldsymbol{z}}_{\downarrow}\right)_{c s j}\right|\right]
\end{aligned}
$$

with $\lambda$ and $\gamma$ some positive hyper-parameters that need to be set. Since OSCAR is a particular case of ordered weighted $\ell_{1}$ norm, its proximity operator is defined in [38, Eq. (24)]. Moreover, the proximity operator is separable across scales and sub-bands, hence its parallelization is easy.

\section{NUMERICAL EXPERIMENTS}

Data acquisition. An ex-vivo baboon brain was scanned on a 7T system (Magnetom Siemens scanner, Erlangen, Germany) using a 32-channel (Nova Medical Inc., Washington, MA, USA) coil $(L=32)$. A modified $2 \mathrm{D} \mathrm{T} 2 *$-weighted GRE sequence was implemented to perform prospective CS using the multi-shot Sparkling trajectories [5] that match a variable density with a polynomial decay $h\left(k_{x}, k_{y}\right)=1 /|k|^{2}$. Shots were generated all together and the sampling scheme was composed of 34 spokes of 3072 samples each (readout of $30.72 \mathrm{~ms}$ per shot and $M=104,448$ samples in total), which leads to an acceleration factor of 15 in time and an under-sampling factor of 2.5. The acquisition parameters were set as follows: $\mathrm{FOV}=200 \times 200 \mathrm{~mm}^{2}$, TR $=550 \mathrm{~ms}$ (for 11 slices), $\mathrm{TE}=30 \mathrm{~ms}, \mathrm{BW}=100 \mathrm{kHz}$ and $\mathrm{FA}=25^{\circ}$ with in-plane resolution of $390 \mu \mathrm{m}$ and $3 \mathrm{~mm}$ slice thickness, and a fully sampled Cartesian reference was acquired using the same sequence parameters (matrix size: $512 \times 512$ ). The corresponding image dimension was $N=512^{2}$. Since the trajectory is non-Cartesian, we used the Nonequispaced FFT [31] to compute $f_{\Omega}$ operator in Eq. (1). We used the Undecimated bi-Orthogonal Wavelet Transform with 4 decomposition scales for $\Psi$ (with $C=4$ and $S_{c}=4, \forall c \in\{1, \ldots, C\}$ and $P_{s}=N, \forall c \in\{1, \ldots, C\}$ and $\left.\forall s \in\left\{1, \ldots, S_{c}\right\}\right)$ as the latter has been shown to increase the image quality in CS reconstruction [39].

For each regularization, the hyper-parameters were tuned so as to maximize sSOS Structural Similarity Metric (SSIM) index [40] using a grid search procedure over a discrete set of values. Note that we did check that the best score was reached in the strict interior of the tested parameter range. All experiments were run on a computer with $128 \mathrm{~GB}$ of RAM and an 8-core $(2.40 \mathrm{GHz})$ Intel Xeon E5-2630 v3 Processor. To have a fair comparison in terms of computing time (Table 2), we disabled the multi-threading acceleration and the number of iterations was set to $K=200$, which appears to be sufficient to reach SSIM stability. Parallelization allowed us to speed up the reconstruction by a factor of 10 using 16 threads for the 
Table 1. Image quality assessment for all regularizers.

\begin{tabular}{|c|c|c|c|}
\cline { 2 - 4 } \multicolumn{1}{c|}{} & SSIM & pSNR $(\mathrm{dB})$ & NRMSE \\
\hline Group-LASSO & 0.864 & 26.92 & 0.254 \\
\hline Sparse group-LASSO & 0.851 & 26.77 & 0.259 \\
\hline OSCAR & $\mathbf{0 . 8 7 5}$ & $\mathbf{3 0 . 4 9}$ & $\mathbf{0 . 1 7 7}$ \\
\hline No regularization & 0.847 & 26.5 & 0.263 \\
\hline$\ell_{1}$-ESPIRiT & 0.874 & 28.32 & 0.238 \\
\hline
\end{tabular}

OSCAR penalization. All the codes have been developed in Python in the open-source PySAP package.

Results. The solution to the $\ell_{1}$-ESPIRiT formulation was taken as the gold standard parallel MRI acceleration techniques. In Tab. 1, the performances of the different penalizations are compared in terms of SSIM, peak SNR (pSNR) and Normalized Root Mean Square Error (NRMSE). The corresponding images are shown in Fig. 1.

The group-LASSO $g_{\mathrm{GL}}$ and its sparse refinement $g_{\mathrm{sGL}}$ provide similar results in terms of image quality. However the sparse groupLASSO requires the setting of an extra hyper-parameter $\mu$ which eventually slows down the tuning step. As suggested in [26], the worse performance of the group-LASSO penalty may be explained in terms of error propagation in the sSOS image. The $\ell_{1}$-ESPIRiT solution seems to better preserve the image structure although we notice the presence of gridding artifacts as confirmed by all image quality scores. One feature of calibrationless reconstruction methods is their ability to recover coil-specific images. Fig 2 compares the SSIM scores across all channels for all penalizations. The groupLASSO and its sparse variation have a reduced dispersion in SSIM while OSCAR extends this variability. In that way, OSCAR better enhances coil-specific images associated with the highest SNR level at the expense of lower SNR channels (see images in Fig. 3).

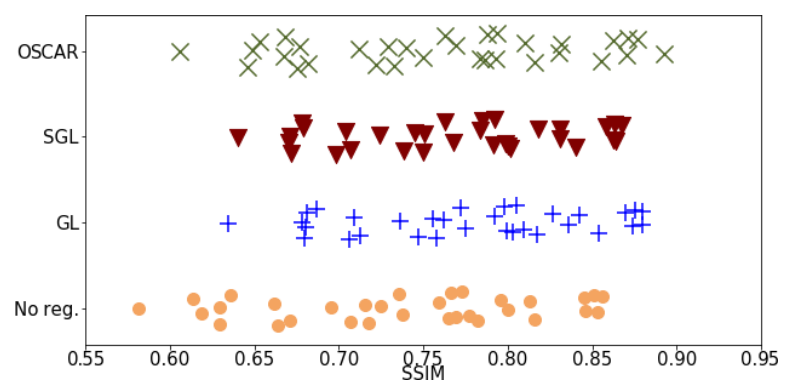

Fig. 2. Assessment of the SSIM score per channel.

Parallelization. One of the limitations of CS reconstruction is the computation time required to recover images especially in the parallel and non-Cartesian imaging paradigm. Hence, it is of critical importance to identify computational bottlenecks and ways of getting rid off them. The computation of the non-Cartesian Fourier operator $f_{\Omega}{ }^{1}$ can be parallelized using the multithreaded acceleration in the pynfft package.

Table 2. Assessment of computation time. To get the total computing time, multiply the time per iteration by $K=200$.

\begin{tabular}{|c|c|c|}
\cline { 2 - 3 } \multicolumn{1}{c|}{} & $\begin{array}{l}\text { Computing time (s) for } \\
\text { proximity operator }\end{array}$ & $\begin{array}{l}\text { Time per } \\
\text { iteration }(\mathrm{s})\end{array}$ \\
\hline Group-LASSO & $\mathbf{7 . 9}$ & 32.9 \\
\hline Sparse group-LASSO & 10.9 & 35.9 \\
\hline OSCAR & 30.9 & 55.9 \\
\hline$\ell_{1}$-ESPIRiT & - & $\mathbf{1 8 . 9}$ \\
\hline
\end{tabular}

\footnotetext{
${ }^{1}$ Its numerical complexity is equal to $O(N(\log (N)+|\log (\epsilon)| M)[31]$, where $\epsilon$ is the desired accuracy
}

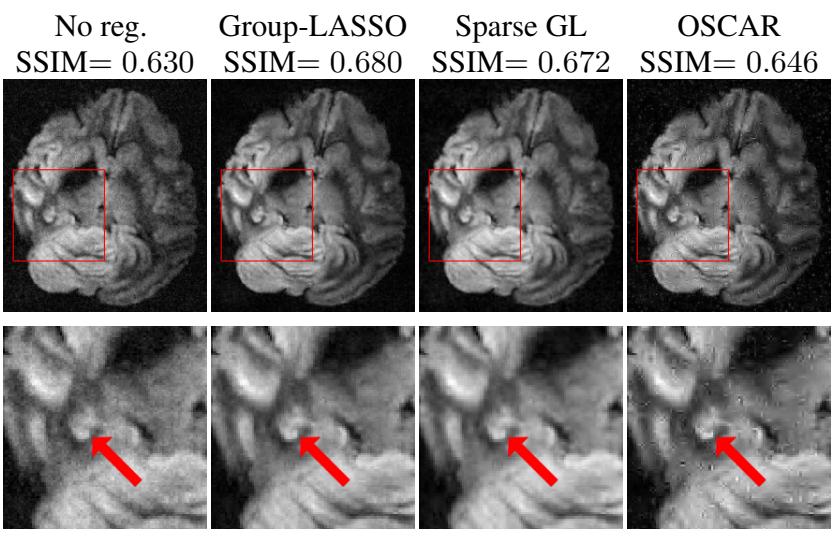

Group-LASSO Sparse GL

OSCAR
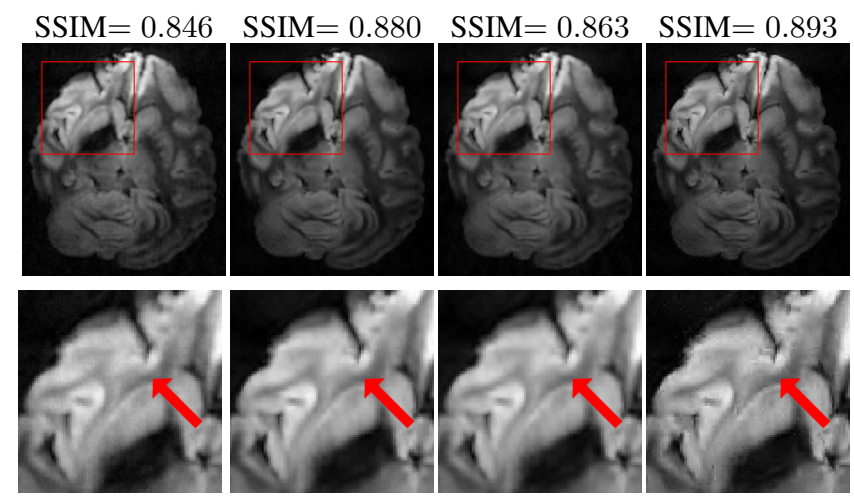

Fig. 3. From left to right, no penalization, group-LASSO solution, sparse group-LASSO and OSCAR solution for two different channels, the first row is a low SNR channel while the second is high SNR.

The different penalizations described in Section 3 have different numerical complexity regarding the calculation of their proximity operator (3): from $O\left(L N_{\Psi}\right)$ for the proximity operator of $g_{\mathrm{GL}}$ and $g_{\mathrm{sGP}}$ to $O\left(N_{\Psi} L \log \left(P_{s} L\right)\right)$ for $g_{\mathrm{OSCAR}}$ one. Hence, this impacts the computing time of these proximity operators as well as the whole reconstruction time as summarized in Tab. 2. In comparison, $\ell_{1}$-ESPIRiT seems less demanding. However, we did not take into account the time required for extracting the sensitivity maps.

\section{CONCLUSION \& DISCUSSION}

In this work, we proposed a new parallel CS-MRI reconstruction algorithm without requiring the extraction of sensitivity maps. This approach can be formulated as a convex optimization problem relying on the OSCAR penalty term. We evaluated the image quality on ex-vivo baboon brain k-space data collected in a prospective and highly accelerated manner at $7 \mathrm{~T}$. We compared OSCAR results to the ones given by the group-LASSO and sparse group-LASSO as well as with the state-of-the-art $\ell 1$-ESPIRiT solution. We demonstrated that one can reconstruct MR images using well suited group sparsity priors without coil sensitivity information, since OSCAR outperforms its competitors. Extended reconstruction tests would be required to assess this observation. In terms of computing time, the reconstruction is more demanding using OSCAR, but a siginificant acceleration can be expected using multi-threading. This might have a significant impact on in-vivo dynamic MRI to mitigate the subjects' motion since an estimation of sensitivity maps is usually required for each scan of the image series. 


\section{REFERENCES}

[1] D. Donoho, “Compressed sensing," IEEE Transactions on Information Theory, vol. 52, no. 4, pp. 1289-1306, 2006.

[2] E. Candès, J. Romberg, and T. Tao, "Robust uncertainty principles: Exact signal reconstruction from highly incomplete frequency information," IEEE Transactions on Information Theory, vol. 52, no. 2, pp. 489-509, 2006.

[3] M. Lustig, D. Donoho, and J. Pauly, "Sparse MRI: The application of compressed sensing for rapid MR imaging," Mag. Res. in Med., vol. 58, no. 6, pp. 1182-1195, 2007.

[4] L. Feng, L. Axel, H. Chandarana, K. Block, D. Sodickson, and R. Otazo, "XD-GRASP: golden-angle radial MRI with reconstruction of extra motion-state dimensions using compressed sensing," Mag. Res. in Med., vol. 75, no. 2, pp. 775-788, 2016.

[5] C. Lazarus, P. Weiss, N. Chauffert, F. Mauconduit, M. Bottlaender, A. Vignaud, and P. Ciuciu, "Sparkling: Novel non-cartesian sampling schemes for accelerated 2D anatomical imaging at 7T using compressed sensing," in 25th annual meeting of the ISMRM, 2017.

[6] L. Kasper, M. Engel, C. Barmet, M. Haeberlin, B. Wilm, B. Dietrich, T. Schmid, S. Gross, D. Brunner, K. Stephan, et al., "Rapid anatomical brain imaging using spiral acquisition and an expanded signal model," Neuroimage, vol. 168, pp. 88-100, 2018.

[7] B. Adcock, A. Hansen, C. Poon, and B. Roman, "Breaking the coherence barrier: A new theory for compressed sensing," in Forum of Mathematics, Sigma. Cambridge University Press, 2017, vol. 5.

[8] N. Chauffert, P. Ciuciu, J. Kahn, and P. Weiss, "Variable density sampling with continuous trajectories. Application to MRI,' SIAM Journal on Imaging Sciences, vol. 7, no. 4, pp. 1962-1992, Nov. 2014.

[9] G. Puy, P. Vandergheynst, and Y. Wiaux, "On variable density compressive sampling," IEEE Signal Processing Letters, vol. 18, no. 10, pp. 595-598, 2011.

[10] C. Lazarus, P. Weiss, A. Vignaud, and P. Ciuciu, "An empirical study of the maximum degree of undersampling in compressed sensing for T2*weighted MRI," Magnetic Resonance Imaging, vol. 53, pp. 112-122, 2018.

[11] P. Roemer, W. Edelstein, C. Hayes, S. Souza, and O. Mueller, "The NMR phased array," Mag. Res. in Med., vol. 16, no. 2, pp. 192-225, 1990.

[12] M. Uecker, T. Hohage, K. Block, and J. Frahm, "Image reconstruction by regularized nonlinear inversionjoint estimation of coil sensitivities and image content," Mag. Res. in Med., vol. 60, no. 3, pp. 674-682, 2008.

[13] M. Lustig and J. Pauly, "Spirit: iterative self-consistent parallel imaging reconstruction from arbitrary k-space," Mag. Res. in Med., vol. 64, no. 2, pp. 457-471, 2010.

[14] J. Haldar, "Low-rank modeling of local $k$-space neighborhoods (LORAKS) for Constrained MRI,' IEEE transactions on Medical Imaging, vol. 33, no. 3, pp. 668-681, 2014.

[15] K. Pruessmann, P. Weiger, M. Scheidegger, and P. Boesiger, "SENSE: sensitivity encoding for fast MRI," Mag. Res. in Med., vol. 42, no. 5, pp. 952-962, 1999.

[16] D. Liang, B. Liu, J. Wang, and L. Ying, "Accelerating SENSE using compressed sensing," Mag. Res. in Med., vol. 62, no. 6, pp. 1574-1584, 2009.

[17] K. Pruessmann, M. Weiger, P. Börnert, and P. Boesiger, "Advances in sensitivity encoding with arbitrary k-space trajectories," Mag. Res. in Med., vol. 46, no. 4, pp. 638-651, 2001.

[18] L. Ying and J. Sheng, "Joint image reconstruction and sensitivity estimation in SENSE (JSENSE)," Mag. Res. in Med., vol. 57, no. 6, pp. 1196-1202, 2007.

[19] H. She, R. Chen, D. Liang, E. DiBella, and L. Ying, "Sparse BLIP: Blind iterative parallel imaging reconstruction using compressed sensing," Mag. Res. in Med., vol. 71, no. 2, pp. 645-660, 2014.
[20] L. Gueddari, C. Lazarus, H. Carrié, A. Vignaud, and P. Ciuciu, "Selfcalibrating nonlinear reconstruction algorithms for variable density sampling and parallel reception MRI," in 10th IEEE SAM workshop, 2018.

[21] M. Uecker, P. Lai, M. Murphy, P. Virtue, M. Elad, J. Pauly, S. Vasanawala, and M. Lustig, "ESPIRiT- an eigenvalue approach to autocalibrating parallel MRI: where SENSE meets GRAPPA," Mag. Res. in Med., vol. 71, no. 3, pp. 990-1001, 2014.

[22] L. Ying and J. Sheng, "Joint image reconstruction and sensitivity estimation in SENSE (JSENSE)," Mag. Res. in Med., vol. 57, no. 6, pp. 1196-1202, 2007.

[23] J. Zhang, C. Liu, and M. Moseley, "Parallel reconstruction using null operations," Mag. Res. in Med., vol. 66, no. 5, pp. 1241-1253, 2011.

[24] A. Majumdar and R. Ward, "Calibration-less multi-coil MR image reconstruction," Mag. Res. in Med., vol. 30, no. 7, pp. 1032-1045, 2012.

[25] J. Trzasko and A. Manduca, "Calibrationless parallel MRI using CLEAR," in Signals, Systems and Computers (ASILOMAR), 2011 Conference Record of the Forty Fifth Asilomar Conference on. IEEE, 2011, pp. 75-79.

[26] I. Chun, B. Adcock, and T. Talavage, "Efficient compressed sensing SENSE pMRI reconstruction with joint sparsity promotion," IEEE transactions on Medical Imaging, vol. 35, no. 1, pp. 354-368, 2016.

[27] Y. Eldar and H. Rauhut, "Average case analysis of multichannel sparse recovery using convex relaxation," IEEE Transactions on Information Theory, vol. 56, no. 1, pp. 505-519, 2010.

[28] P. Boufounos, G. Kutyniok, and H. Rauhut, "Sparse recovery from combined fusion frame measurements," IEEE Transactions on Information Theory, vol. 57, no. 6, pp. 3864-3876, 2011.

[29] J. Friedman, T. Hastie, and R. Tibshirani, "A note on the group lasso and a sparse group lasso," arXiv preprint arXiv:1001.0736, 2010.

[30] H. Bondell and B. Reich, "Simultaneous regression shrinkage, variable selection, and supervised clustering of predictors with OSCAR," Biometrics, vol. 64, no. 1, pp. 115-123, 2008.

[31] J. Keiner, S. Kunis, and D. Potts, "Using NFFT 3-a software library for various nonequispaced fast fourier transforms," ACM Transactions on Mathematical Software (TOMS), vol. 36, no. 4, pp. 19, 2009.

[32] J. Fessler and B. Sutton, "Nonuniform fast fourier transforms using min-max interpolation," IEEE Transactions on Signal Processing, vol. 51, no. 2, pp. 560-574, Feb 2003.

[33] L. Condat, "A primal-dual splitting method for convex optimization involving lipschitzian, proximable and linear composite terms," Journal of Optimization Theory and Applications, vol. 158, no. 2, pp. 460-479, Aug 2013.

[34] B. Vũ, "A splitting algorithm for dual monotone inclusions involving cocoercive operators," Advances in Computational Mathematics, vol. 38, no. 3, pp. 667-681, Apr 2013.

[35] N. Komodakis and J.-C. Pesquet, "Playing with duality: An overview of recent primal? dual approaches for solving large-scale optimization problems," IEEE Signal Processing Magazine, vol. 32, no. 6, pp. 3154, 2015.

[36] W. Press, S. Teukolsky, W. Vetterling, and B. Flannery, Numerical Recipes 3rd Edition: The Art of Scientific Computing, Cambridge University Press, New York, NY, USA, 3 edition, 2007.

[37] N. Pustelnik, A. Benazza-Benhayia, Y. Zheng, and J.-C. Pesquet, Wavelet-Based Image Deconvolution and Reconstruction, pp. 1-34, American Cancer Society, 2016.

[38] Z. X. and M. Figueiredo, "The ordered weighted 11 norm: Atomic formulation, projections, and algorithms," arXiv preprint arXiv:1409.4271, 2014.

[39] H. Cherkaoui, L. Gueddari, C. Lazarus, A. Grigis, F. Poupon, A. Vignaud, S. Farrens, J. Starck, and P. Ciuciu, "Analysis vs synthesis-based regularization for combined compressed sensing and parallel MRI reconstruction at 7 tesla," in 26th European Signal Processing Conference (EUSIPCO 2018), 2018.

[40] Z. Wang, A. Bovik, H. Sheikh, and E. Simoncelli, "Image quality assessment: from error visibility to structural similarity," IEEE transactions on Image Processing, vol. 13, no. 4, pp. 600-612, 2004. 